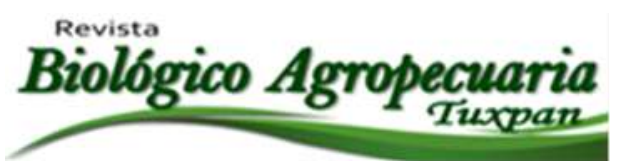

\title{
Plantas alimenticias no convencionales de una zona periurbana de Coatepec, Veracruz
}

Food plants nonconvential from the peri-urban zone of Coatepec, Veracruz.

Lozada García Dulce María ${ }^{1 凶}$, Garibay Pardo Leticia ${ }^{1}$, Cházaro Basáñez Miguel de Jesús ${ }^{1}$, Lozada García José Armando ${ }^{1}$ y Domínguez González Nancy ${ }^{2}$

${ }^{1}$ Facultad de Biología Xalapa y ${ }^{2}$ Facultad de Ciencias Agrícolas Xalapa, Universidad Veracruzana, Circuito Universitario Gonzalo Aguirre Beltrán s/n. Colonia Zona Universitaria, Xalapa, Veracruz. C.P. 91050.

${ }^{凶}$ Autor de correspondencia: dulcema_2@hotmail.com

Recibido: $13 / 07 / 2018$

Aceptado: 12/09/2018

\section{RESUMEN}

Entre los recursos naturales que el hombre ha utilizado, existen las plantas alimenticias no convencionales (PANC), es decir aquellas plantas que no se cultivan o comercializan en gran cantidad, están adecuadas al medio local facilitando su cultivo e inclusive pueden ser plantas olvidadas. Cuya utilización se está desaprovechando, ya que se están perdiendo los conocimientos tradicionales acerca del uso que tienen aunado al deterioro de la vegetación primaria. Ante esta situación se planteó como objetivo de este trabajo el documentar los conocimientos tradicionales sobre las PANC en una zona periurbana de Coatepec, Ver, con lo que se busca mantener el aprovechamiento racional de éste recurso biológico. En un primer momento, para recabar la información se identificaron a informantes clave y se les aplicaron entrevistas semiestructuradas; posteriormente, se realizaron encuestas a la población del área de estudio para determinar el grado de conocimiento que tenían sobre estas plantas. Se registraron 34 especies de PANC, distribuidas en 22 familias, siendo las más representativas la familia Solanaceae con siete especies y Asteraceae con cuatro. Las personas concuerdan que este tipo de plantas son de gran importancia ya que fueron y en algunos casos siguen siendo parte de su alimentación, se reconoce que el conocimiento tradicional y uso de las mismas se resguarda en las personas mayores, por lo que es importante rescatar estos saberes y sobre todo compartirlos con las generaciones más jóvenes para que las sigan aprovechando y no se pierda su uso.

Palabras clave: Conocimiento tradicional, etnobotánica, periurbano, informantes clave.

\begin{abstract}
ABSTRAC
Among the natural resources that man has used, there are nonconventional food plants ((NCEP), that is to say those plants that are not cultivated or marketed in large quantity, are suitable to the local environment facilitating their cultivation and even can be forgotten plants. The use of which is being wasted as traditional knowledge about the use of the primary vegetation is being lost. Faced with this situation was raised as the objective of this work to document the traditional knowledge on the NCEP in a peri-urban area (Coatepec, Veracruz), which seeks to maintain the rational use of this biological resource. Initially, to collect information, key informants were identified and semi-structured interviews were applied; subsequently, surveys were conducted on the population of the study area to
\end{abstract}


determine the degree of knowledge they had on these plants. We recorded 34 species of NCEP, distributed in 22 families, the most representative being the family Solanaceae with seven species and Asteraceae with four. People agree that these types of plants are of great importance because they were and in some cases remain part of their food, it is recognized that traditional knowledge and use of them is protected in older people, so it is important rescuing these knowledge and above all share them with younger generations to continue taking advantage and not lose their use.

Keywords: Traditional knowledge, ethnobotany, peri-urban, key informants.

\section{INTRODUCCIÓN}

En México existe un diverso patrimonio etnobotánico debido a la relación que las comunidades mantienen con su entorno natural, es uno de los países con mayor diversidad biológica y se estima que existen unas 7000 especies de plantas útiles (Caballero y Cortés, 2001). A su vez, el estado de Veracruz se encuentra en tercer lugar en biodiversidad después de Oaxaca y Chiapas, y es uno de los más ricos en flora, ya que en él confluyen dos regiones biogeográficas del continente americano, la Neártica y la Neotropical (Luna, 1997).

Dentro de esta diversidad, existen las plantas alimenticias no convencionales (PANC), es decir aquellas plantas que no se cultivan o comercializan en gran cantidad e inclusive pueden ser plantas olvidadas o no reconocidas como plantas comestibles por la totalidad de la población según Kinupp et al. (2016), cuya utilización se está desaprovechando (Chávez, 2010), debido a los cambios de estilo de alimentación impuestos a través de un mercado globalizado y a la falta de interés de las generaciones actuales de continuar con este legado cultural (Ysunza et al., 2011). Así mismo, la vegetación primaria se ha ido transformando y deteriorando a consecuencia de distintos factores provocados por el hombre, como son el cambio de uso del suelo y el crecimiento de la mancha urbana (Luna, 1997), lo que repercute en una pérdida significativa de biodiversidad, y entre ella las PANC. Perdiendo no sólo el recurso natural, sino también los conocimientos tradicionales generados a través de prácticas empíricas de las personas, en interacción con el medio ambiente (Kinupp et al., 2016).

Por tal motivo se tuvo como objetivo principal documentar los conocimientos tradicionales que las personas tienen del uso de las plantas alimenticias no convencionales en una zona periurbana de Coatepec en el estado de Veracruz, México.

\section{MATERIALES Y MÉTODOS}

Coatepec se encuentra situada en la zona central montañosa del estado de Veracruz, México, en las estribaciones orientales del Cofre de Perote, a una altura de $1200 \mathrm{msnm}$ con una superficie total de $255.81 \mathrm{~km}^{2}$, presenta remanentes de vegetación primaria de bosque mesófilo de montaña y bosque de coníferas (INEGI, 2015). En primer lugar y al tratarse de una zona amplia geográficamente, se delimitó el área de estudio, a dos colonias de la periferia de la ciudad de Coatepec, las cuales colindan con zonas de vegetación de relativa importancia.

Con el fin de recabar información sobre las PANC, se identificaron informantes clave, personas mayores de 50 años, que tuvieran un amplio conocimiento sobre la utilidad de diferentes plantas, adquirido a través de sus experiencias de vida. Se elaboró un primer instrumento que se aplicó en forma de 
entrevista semiestructurada, utilizando la técnica de cadena por referencia (Mendieta, 2015). Posteriormente se llevó a cabo la colecta de ejemplares botánicos, para su identificación taxonómica. La información obtenida se organizó en una base de datos, después se realizaron encuestas a la población en general de las colonias de la periferia donde se trabajó, para conocer su grado de conocimiento sobre las PANC reportadas previamente; la información obtenida fue analizada considerando dos índices etnobotánicos; Frecuencia Relativa de Citaciones (RFC) que supone la cantidad de encuestados que reconocieron una planta determinada como PANC, determinándose de la siguiente manera: $\mathrm{RFC}=\mathrm{N} / \mathrm{Ni}$, donde $\mathrm{N}$ es el número de encuestados que citaron una planta alimenticia y $\mathrm{Ni}$ es el número total de encuestados, un $\mathrm{RFC}=1$ indica que todos los encuestados identificaron la planta como alimenticia no convencional y un $\mathrm{RFC}=0$ indica que ningún encuestado la identifica como una planta alimenticia (Tardío y Pardo-de-Santayana, 2008). El segundo Índice es el de Riqueza, que es la riqueza de conocimiento que tiene una persona sobre las posibilidades de uso de la flora local, donde se utilizó la siguiente fórmula: $\mathrm{RQZ}=\sum \mathrm{EU}$ Valor / EU Máximo, en donde: RQZ es la riqueza de conocimiento que tiene una persona de las especies útiles, EU es el número de especies útiles registradas por una persona y el Valor EU Máximo es el total de especies útiles reportadas en la región de todas las personas participantes del estudio (Castellanos, 2011), también se analizó cada pregunta realizada en las encuestas.

\section{RESULTADOS Y DISCUSIÓN}

La identificación de los informantes clave se llevó a cabo en un periodo que abarcó desde finales del mes de septiembre, octubre y parte de noviembre del 2017. Se logró el registro de seis personas, con un rango de edad que abarca de los 62 a los 76 años; de acuerdo a las vivencias y experiencias de estas personas, concuerdan que las PANC son de gran importancia ya que fueron, y en algunos casos siguen siendo parte de su alimentación.

A partir de los datos aportados, se logró registrar 34 especies de PANC; distribuidas en 22 familias, aquella con mayor número de especies fue la familia Solanaceae con siete especies, es una de las familias de fanerógamas más extensa que se distribuye en todo el mundo y de importancia dentro de la alimentación humana, seguida de Asteraceae con cuatro especies, Melastomataceae y Commelinaceae con dos especies y el resto con una especie (Figura

$1)$.

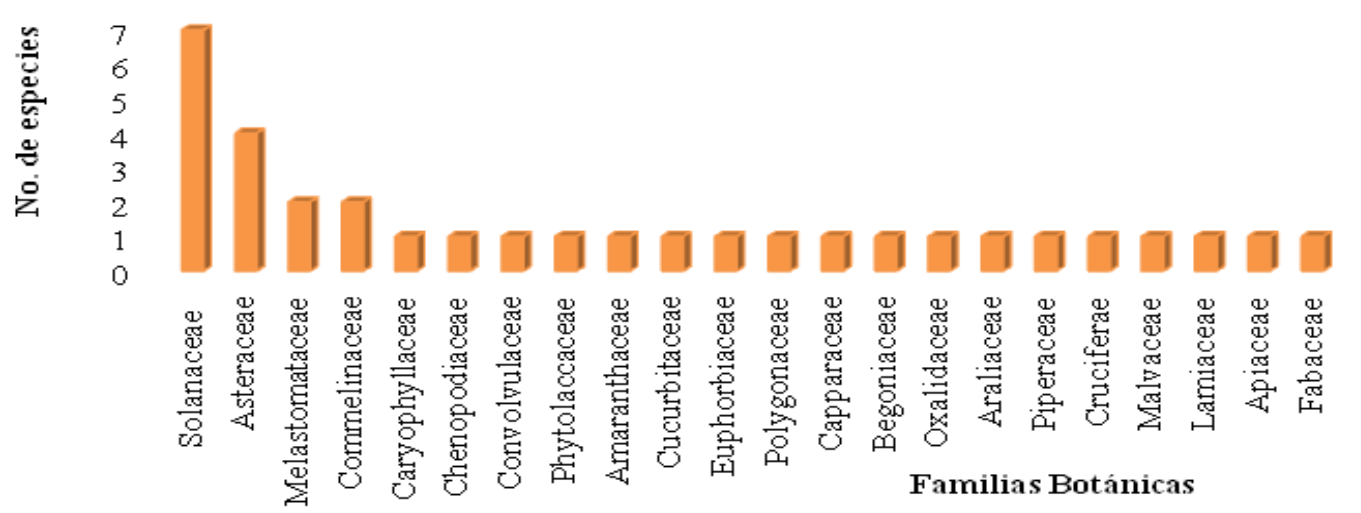

Figura 1. Diversidad de especies reportadas por familia botánica de PANC 
En función de las preguntas realizadas en las encuestas, las hojas de las plantas son la parte más utilizada, seguido por el porcentaje de hojas y tallo, es decir, cuando la planta está tierna ocupan no sólo las hojas sino también el tallo (Figura 2). En la figura 3 se observa que la mayoría de las personas las recolectan en el campo; otra manera de adquirirlas, aunque en menor porcentaje, es la colecta en sus propios patios y muy pocas personas las compran, por tener la característica de no ser tan comunes y por ende no se suelen cultivar para su consumo.

Las personas mencionaron que consumían algunas de las PANC por su sabor, continuando el consumo por costumbre, ya que formaban parte de un hábito alimenticio transmitido a través de las generaciones (Figura
4). Además, comentaron que sus padres eran quienes les dieron a conocer la utilidad de las plantas, al ser parte de sus alimentos. Los amigos y los abuelos también son participes de la transmisión de la utilidad ya que en el caso de éstos últimos, su alimentación estaba basada en plantas (Figura 5).

La transmisión de conocimientos es principalmente de padres a hijos, aunque también se comparten a través de la convivencia entre amigos, ya sea en sus lugares de trabajo o de esparcimientos cotidianos, así mismo, representó mayoría el hecho de que nunca han transmitido estos saberes a nadie, porque las menosprecian o las consideran solo un alimento para animales (Figura 6).

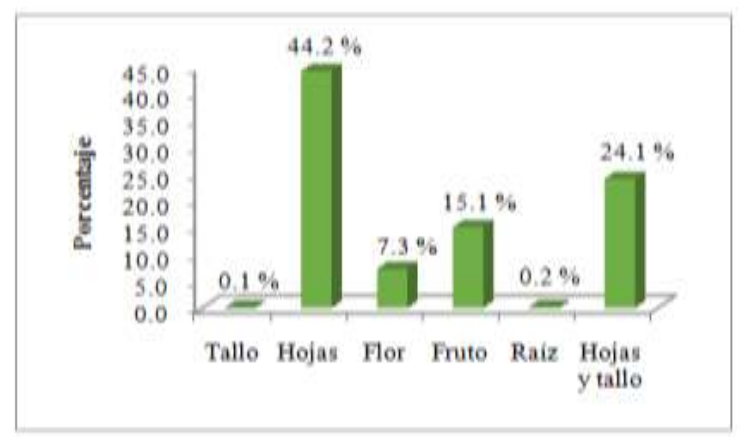

Figura 2. Distribución de la parte utilizada de la planta.

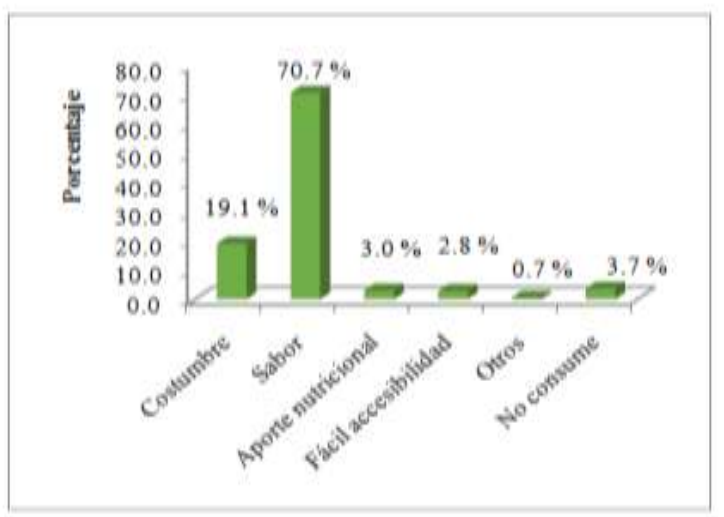

Figura 4. Distribución del motivo de su consumo

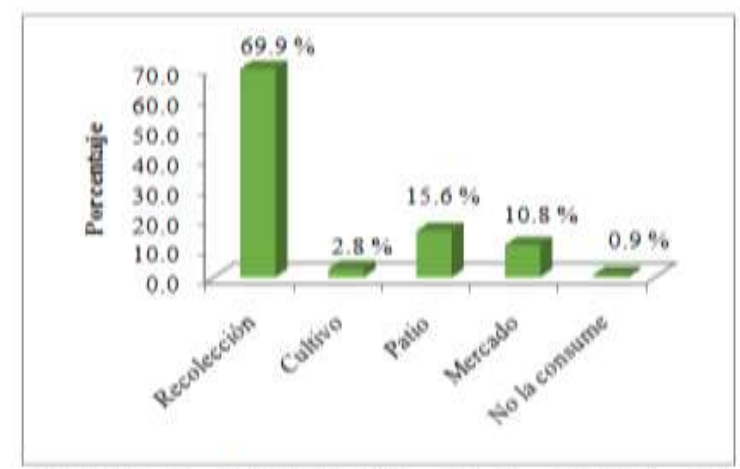

Figura 3. Distribución de la manera de adquirir la planta alimenticia.

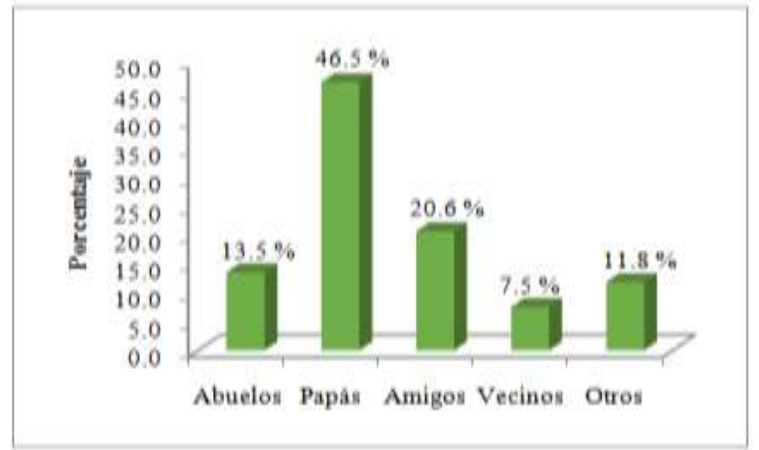

Figura 5. Distribución de quiénes transmiten más el conocimiento de la utilidad de la planta 
Índice de Frecuencia Relativa de Citaciones (RFC)

Con respecto al índice de Frecuencia Relativa de Citaciones (RFC), los resultados muestran (Tabla 1) que las tres especies más mencionadas fueron el tomate citlali (Lycopersicon esculentum) con RFC de 1, usualmente usado y conocido por su peculiar sabor en la preparación de salsas. El tomate citlali no es una especie cultivada como tal, pero es común verlo en algunos cultivos de manera silvestre, se le recolecta para su consumo y muy poco es ofertado en el mercado local. El cilantro de monte (Peperonia peltilimba) planta que crece de manera silvestre, aunque también se puede encontraren algunos patios, es utilizada como condimento, aunque también se comercializa poco. La hierba mora (Solanum americanum) se considera además de comestible como una planta medicinal, por tal motivo se consume.

Tabla 1. Índice de Frecuencia relativa de citaciones (RFC)

\begin{tabular}{c|cccc} 
NOMBRE COMUN & NOMBRE CIENTIFICO & FRECUENCIA & RFC & PORCENTAJE (\%) \\
\hline Citlali & Lycopersicon esculentum & 36 & 1 & 100 \\
Cilantro de Monte & Peperonia peltilimba & 35 & 0.97 & 97.22 \\
Hierba Mora & Solanum americanum & 32 & 0.89 & 88.89
\end{tabular}

\section{Análisis de la Riqueza de Conocimiento (RQZ)}

Los datos obtenidos en este índice nos indican que de las 36 encuestas que se realizaron, las personas en un rango de edad de 20 a 40 años tienen un conocimiento menor ( $28 \%)$, por lo contrario, las personas mayores de 40 años mostraron mayor conocimiento de la utilidad de éstas plantas (45\%)al igual que lo reportado por Hurtado et al. (2006); esto constata que el conocimiento sobre el uso de este tipo de plantas se encuentra principalmente en las personas mayores. En la Tabla 2 se visualiza parte de la información comparando ambos rangos de edad utilizados en este índice.

Tabla 2. Índice de Riqueza de Conocimiento (RQZ)

\begin{tabular}{|c|c|c|c|c|c|}
\hline \multicolumn{6}{|c|}{ DENTRO DEL RANGO DE EDAD DE 20 A 40 AÑOS DE EDAD } \\
\hline EDAD & SEXO & OCUPACIÓN & $\begin{array}{c}\text { PLANTAS } \\
\text { MENCIONADAS }\end{array}$ & RQZ & $\begin{array}{c}\text { PORCENTAJE } \\
(\%)\end{array}$ \\
\hline 22 & $\mathrm{~F}$ & Estudiante & 12 & 0.35 & 5 \\
\hline 23 & $\mathrm{~F}$ & Estudiante & 6 & 0.18 & 18 \\
\hline 26 & $\mathrm{~F}$ & Ama de casa & 3 & 0.09 & 9 \\
\hline 28 & M & Empleado & 10 & 0.29 & 29 \\
\hline 28 & $\mathrm{~F}$ & Ama de casa & 9 & 0.26 & 26 \\
\hline \multicolumn{6}{|c|}{ DENTRO DEL RANGO DE EDAD DE 40 AÑOS EN ADELANTE } \\
\hline 67 & M & Jardinero & 18 & 0.53 & 53 \\
\hline 68 & M & Zapatero & 17 & 0.50 & 50 \\
\hline 69 & $\mathrm{~F}$ & Ama de casa & 20 & 0.59 & 59 \\
\hline 70 & M & Campesino & 15 & 0.44 & 44 \\
\hline 74 & $\mathrm{~F}$ & Ama de casa & 17 & 0.50 & 50 \\
\hline
\end{tabular}




\section{CONCLUSIONES}

Se obtuvo un registro de 34 plantas alimenticias no convencionales, distribuidas en 22 familias identificadas botánicamente, se considera una cantidad importante debido a que el estudio realizado se delimitó en un marco geográfico relativamente pequeño; la familia más representativa fue la Solanaceae.

La mayoría de las PANC se consiguen mediante recolección y algunas por cultivo de traspatio. Se reconoce que las hojas son la parte de mayor consumo, y se consumen principalmente por su sabor y por costumbre, haciéndose notar que los conocimientos de su utilidad se pasan de generación en generación.

Respecto al índice de frecuencia relativa de citaciones (RFC),la especie más reconocidafueel Citlali (Lycopersicon esculentum). El índice de Riqueza (RQZ) que tuvieron las personas sobre las PANC, muestra que las personas mayores de 40 años reconocieron un mayor número de plantas. Se reconoce que el conocimiento de la utilidad de estas plantas se resguarda en las personas mayores de 50 años, por lo que es importante rescatar estos saberes y sobre todo compartirlos con las generaciones futuras para que se sigan aprovechando de una manera racional.

\section{LITERATURA CITADA}

Caballero, J. y Cortés, L. 2001. Percepción, uso y manejo tradicional de los recursos vegetales en México. Plantas cultura y Sociedad. Universidad Autónoma Metropolitana-SEMARNAT. México. pág. 79-100.
Castellanos, L. 2011. Conocimiento etnobotánico, patrones de uso y manejo de plantas útiles en la cuenca del río Cane-Iguaque (Boyocá-Colombia); una aproximación desde los sistemas de uso de la biodiversidad. Ambiente \& Sociedades (6): 45-75. https://doi.org/10.1590/S1414-753X2011 000100004

Chávez, E. 2010. Plantas comestibles no convencionales en Chiapas. Tuxtla Gutiérrez, Chiapas, México: Editorial. Universidad de Ciencias y Artes de Chiapas. pag. 138.

Hurtado, N., Rodríguez, C. y Aguilar, A. 2006. Estudio Cualitativo y Cuantitativo de la flora medicinal del municipio de Copándaro de Galeana, Michoacán, México. Polibotánica. Núm. 22. pp. 2150.

INEGI. 2015. Coatepec. Estado de Veracruz de Ignacio de la llave. Cuaderno Estadístico Municipal. Edición 2015.

Kinupp, V., Jiménez, A. y Vela, M. 2016. Las Plantas Alimenticias No Convencionales (PANC) como estrategia de Resiliencia en la Amazonia. Agroecología. Recuperado de:

https://www.agroecologia.net/recursos/p ublicaciones/actas/cd-actasxcongresoseae/actas/comunicaciones/93 -plantas-jimenez.pdf.

Luna, V. 1997. Estudio de vegetación y flora del municipio de Coatepec, Ver. Tesis. Facultad de Biología. Universidad Veracruzana. Xalapa, Ver.

Mendieta, G. 2015. Informantes y muestreo en investigación cualitativa. Investigaciones Andina, vol. 17, núm. 30, abril-septiembre. 
Tardío, J. y Pardo-de-Santayana, M. 2008.

Cultural Importance Indices: A

Comparative Analysis Based on the

Useful Wild Plants of Southern

Cantabria (Northern Spain). Economic

Botany 62: 24-39.

https://doi.org/10.1007/s12231-007-9004

$-5$

Ysunza, A., Urdanivia, C., López, L. y Martínez, M. E. 2011. Plantas comestibles no convencionales: el conocimiento que los campesinos mexicanos tienen, pero que no saben que tienen.Saberes colectivos y diálogo de saberes en México. Cuernavaca, Mor: CRIM. UNAM. INAH.UIA. FONCICYT. paga 345.

Copyright (c) 2018 Dulce Maria Lozada G arcia, Leticia G aribay Pardo, Miguel de Jesús Cházaro Basáñez, José Armando Lozada Garcia y Nancy Dominguez González

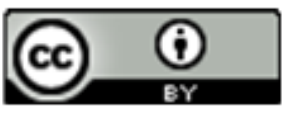

E ste tex to está protegido por una licencia CreativeCommons 4.0

Usted es libre para Compartir —copiar y redistribuir el $\mathrm{m}$ aterial en cualquier medio o formato-y Adaptar el documento —remezclar, transformar y crear a partir del material- para cualquier propósito, incluso para fines comerciales, siempre que cumpla la condición de:

Atribución: Usted debe dar crédito a la obra original de manera adecuada, proporcionar un enlace a la licencia, e in dicar si se han realizado cambios. Puede hacerlo en cualquier forma razonable, pero no de forma tal que sugiera que tiene el apoyo del licenciante olo recibe por el uso que hace de la obra.

Resumenclelicencia - Textocompletodelalicencia 